\title{
Skin cancer detection by spectroscopic oblique-incidence reflectometry: classification and physiological origins
}

\author{
Alejandro Garcia-Uribe, Nasser Kehtarnavaz, Guillermo Marquez, Victor Prieto, \\ Madeleine Duvic, and Lihong V. Wang
}

\begin{abstract}
Data obtained from 102 skin lesions in vivo by spectroscopic oblique-incidence reflectometry were analyzed. The participating physicians initially divided the skin lesions into two visually distinguishable groups based on the lesions' melanocytic conditions. Group 1 consisted of the following two cancerous and benign subgroups: (1) basal cell carcinomas and squamous cell carcinomas and (2) benign actinic keratoses, seborrheic keratoses, and warts. Group 2 consisted of (1) dysplastic nevi and (2) benign common nevi. For each group, a bootstrap-based Bayes classifier was designed to separate the benign from the dysplastic or cancerous tissues. A genetic algorithm was then used to obtain the most effective combination of spatiospectral features for each classifier. The classifiers, tested with prospective blind studies, reached statistical accuracies of $100 \%$ and $95 \%$ for groups 1 and 2 , respectively. Properties that related to cell-nuclear size, to the concentration of oxyhemoglobin, and to the concentration of deoxyhemoglobin as well as the derived concentration of total hemoglobin and oxygen saturation were defined to explain the origins of the classification outcomes. (C) 2004 Optical Society of America
\end{abstract}

OCIS codes: $170.3890,300.0300,100.2960,290.0290$.

\section{Introduction}

Skin cancer is the most common of all cancers; it accounts for nearly $40 \%$ of all cancer cases, ${ }^{1}$ and its incidence is increasing. Suspicious skin lesions are often biopsied, a procedure that is unpleasant for the patient and slow to yield diagnostic results. In addition, the rate of unnecessary biopsies is high. In our data set, $52 \%$ of the biopsies were unnecessary. Hence there is a need for a fast, accurate, and noninvasive detection method to lower the number of unnecessary biopsies as well as to minimize false negatives that are missed by dermatologists.

A. Garcia-Uribe, Guillermo Marquez, and L. V. Wang (lwang@tamu.edu) are with Texas A\&M University, College Station, Texas 77843-3120. A. Garcia-Uribe and L. V. Wang are with the Department of Electrical Engineering and G. Marquez and L. V. Wang are with the Department of Biomedical Engineering. N. Kehtarnavaz is with the Department of Electrical Engineering, University of Texas at Dallas, Richardson, Texas 75080. V. Prieto and M. Duvic are with the University of Texas M. D. Anderson Cancer Center, Houston, Texas 77030.

Received 7 November 2003; revised manuscript received 6 February 2004; accepted 18 February 2004.

0003-6935/04/132643-08 $\$ 15.00 / 0$

(C) 2004 Optical Society of America
Previous works related to the classification of cellular abnormalities by spectroscopy are summarized briefly here. Ganster et al. ${ }^{2}$ developed a system for the computerized analysis of images obtained from epiluminescence microscopy to enhance the early recognition of malignant melanoma. The features that they focused on included shape and radiometric features as well as local and global parameters. Wallace et $a l .{ }^{3}$ studied the optical reflectance characteristics of pigmented skin lesions and evaluated the potential of the technique for distinguishing between malignant melanoma and benign pigmented skin lesions. Backman et al. ${ }^{4}$ showed results that suggested that light scattering might be used to identify epithelial precancerous lesions and preinvasive cancers throughout the body through the use of reduced scattering coefficient spectra. Cooney et al. ${ }^{5}$ investigated the spectral differences in the nearinfrared range among malignant, benign, and healthy oral tissue, using a Fourier-transform spectrometer. Roning and Riech ${ }^{6}$ developed a skin cancer detection system to detect the early occurrence of melanoma. Using a computer-vision system, they screened the body of a patient at regular intervals for changes or new lesions. To detect changes suggestive of melanoma, they compared repeated registra- 


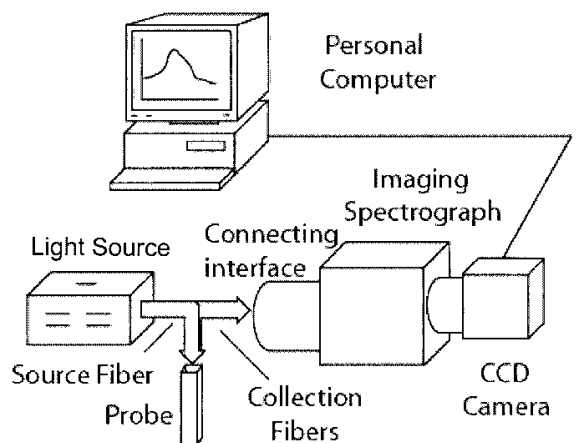

(a)

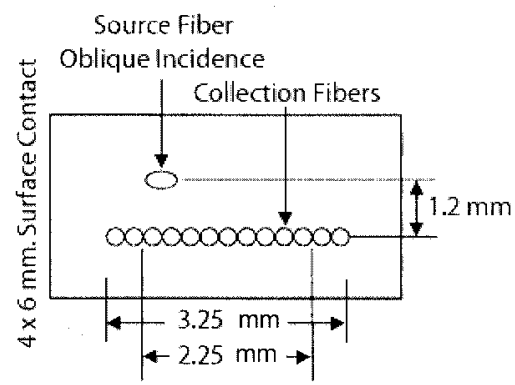

(b)

Fig. 1. (a) Schematic of SOIR. (b) Configuration of the opticalfiber probe.

tions of skin images for alterations in size, shape, and color. In Ref. 7, Mehrubeoglu et al. described using spectroscopic oblique-incidence reflectometry (SOIR) to classify skin lesions.

In an effort to improve on the results reported in Ref. 7 , in the present study we examined new image features that were based on a continuous wavelet transform and were selected by use of a genetic algorithm. Further, from the study we determined how those physiological parameters that can be measured by SOIR - the oxygen saturation of hemoglobin, the concentration of total hemoglobin, and the average size of the cell nuclei of the lesions-are related to skin lesions.

In Sections 2-4 we present a description of how wavelet-based spectral features can be used to distinguish different types of skin lesion. In Sections 5 and 6 several physiological parameters that provide a link between the extracted features and the physiological origins of the skin lesions are derived from the optical properties.

\section{Overview of Spectral Imaging Setup}

The SOIR system is illustrated in Fig. $1 .^{8}$ White light from a halogen lamp (Dolan-Jenner Industries, FiberLite High Intensity Illuminator 170-D) is coupled to a single optical fiber (source fiber) that delivers light to the skin area of interest. A linear array of 13 low-OH (low-loss) fibers (200- $\mu$ m diameter) collects the diffuse reflectance from the skin. The output of the connecting interface is placed in the object plane of the imaging spectrograph (Oriel, Multispec 257).

With the probe surface placed perpendicularly to

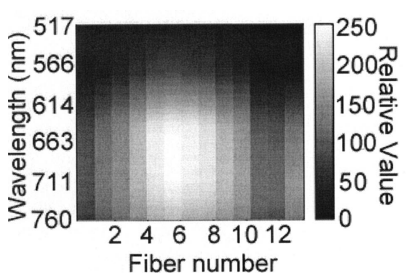

Actinic keratosis

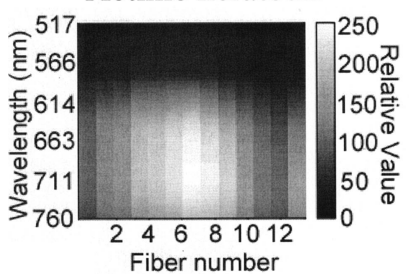

Common nevi

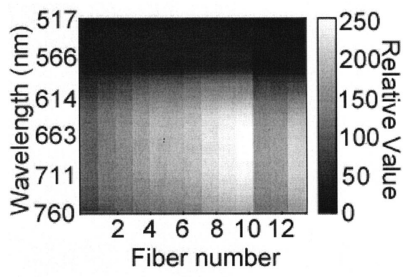

Squamous cell carcinoma

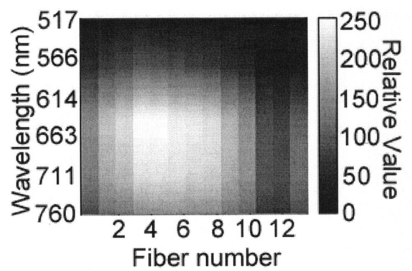

Seborrheic keratosis

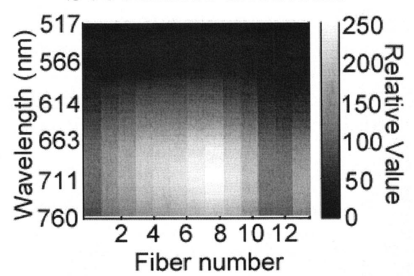

Basal cell carcinoma

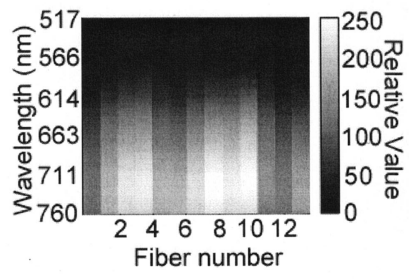

Dysplastic nevi

Fig. 2. Sample SOIR images: intensity of light is represented by different shades. Vertical axis, wavelengths in nanometers; horizontal axis, probe fiber number.

the surface of the skin, the source fiber is oriented at a 45-deg angle of incidence. The probe is positioned on the skin surface with minimal pressure to ensure good contact between the probe and the skin surface for data acquisition. The spectrograph generates an optical spectrum for each optical fiber and projects the spectra onto an 18-bit CCD camera (Princeton Instruments, Inc., 1530P). Data are collected in the range $517-760 \mathrm{~nm}$; ultraviolet light is not desirable because it is unsafe for the patients, nor is infrared light because it is less sensitive to hemoglobin. The chosen visible spectral range can be obtained from inexpensive light sources and is able to quantify the three physiological parameters that we explored.

The collected information is then displayed as a spatiospectral image. Figure 2 shows a typical image obtained by the SOIR device. The horizontal dimension in this image indicates the fiber number of the probe. The vertical dimension represents the spectral distribution of the light from each fiber. A personal computer records the spatiospectral images automatically.

Clinical testing was performed at the University of Texas M. D. Anderson Cancer Center, Houston, Texas. A total of 102 lesions were measured with the ambient light off. Suspicious lesions were divided into two groups based on their visual appearance. However, the dermatologists had difficulty in visually distinguishing the benign lesions from the dysplastic or cancerous lesions within each group. Group 1 consisted of the following two subgroups: (1) cancerous basal cell carcinomas (BCC) and squa- 
mous cell carcinomas (SCC) and (2) benign actinic keratoses (AK), seborrheic keratoses (SK), and warts (W). Group 2 consisted of the following two subgroups: (1) dysplastic nevi (DN) and (2) benign common nevi (CN).

For the design of the group 1 classifier, 220 spatiospectral images were collected from 23 skin lesions. A maximum of five images were collected from each lesion site and a maximum of five images from the adjacent normal skin. The histology identified 8 of the 23 lesions as carcinomas (SCC and BCC) and 15 as benign lesions (AK, SK, or W). For the design of the group 2 classifier, 364 spatiospectral images were collected from 42 lesions and comprised 182 spatiospectral images from lesions and, for self-referencing, the same number of images from the adjacent normal skin. In this group, 25 of the 42 lesions were premalignant lesions (DN) and 17 were normal melanocytic nevi $(\mathrm{CN})$. It is worth pointing out that the collected database did not capture any melanoma cases because of the low clinical occurrence of such cases.

\section{Image Preprocessing}

Before each image was stored, background subtraction was performed to remove darkroom camera system noise. The optical-fiber bundle occupied 3.25 $\mathrm{mm}$ on the contact surface [Fig. 1(b)]. With lesions smaller than this size, the outer fibers collected diffusely reflected light from the surrounding healthy tissue. For this reason the data from the first two and the last two fibers were removed to permit consistent analysis for all the collected images, thus reducing the effective probe size to $2.25 \mathrm{~mm}$. A running-average filter with a window size of five data points was applied to the spectra collected from each fiber to reduce noise. The resultant images were then calibrated against the transmission factors of each fiber; the fact that each fiber possessed different transmission properties was taken into account. Finally, the images were rescaled from 0 to 255 while floating-point precision was held constant.

\section{Data Analysis}

A. Extraction and Selection of Wavelet-Based Features

Reference 7 reported that a number of features were obtained that provided a good differentiation between benign and cancerous or dysplastic tissues. The spatiospectral features used in the present study, however, were found to be more effective than those reported in Ref. 7. These features are based on the continuous wavelet transform (CWT).

Here we present a brief description of the CWT to provide a clearer understanding of the features to be presented in Tables 1 and 2 below. For more details about wavelets the reader is referred to the many references on wavelets, for example, Ref. 9. The CWT of function $f(t)$ with respect to analyzing wavelet function $\psi$ is defined as follows ${ }^{9}$ :

$$
W f_{\psi}(b, a)=\frac{1}{\sqrt{a}} \int_{-\infty}^{\infty} f(t) \psi\left(\frac{t-b}{a}\right) \mathrm{d} t, \quad a>0 .
$$

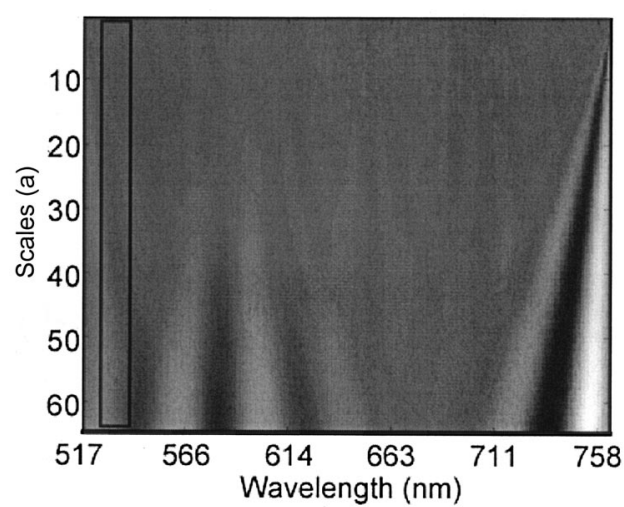

Fig. 3. Example of a Morlet wavelet and 64 scaling functions. Each CWT feature is extracted from a portion of this twodimensional plot.

Parameter $b$ is called the translation parameter; it indicates the position or location of the wavelet function. Parameter $a$ is called the dilatation parameter. The inverse of this parameter, $1 / a$, can be regarded as a measure of frequency. By changing $b$ and $a$ one can present transform $W f_{\psi}(b, a)$ as a socalled two-dimensional time-scale or position-scale plot. Each CWT feature is extracted from a portion of this two-dimensional plot. Figure 3 illustrates an example of the corresponding position-scale plot with the Morlet wavelet. 9

A total of 432 features reflecting the first, second, third, and fourth moments of the CWT were extracted from the spectra collected by our device. Furthermore, the same features were extracted from the images collected from the neighboring healthy tissues. These feature values were used as references (controls) to eliminate biological variations. The following three healthy-tissue normalization schemes were considered for each of these features: $\mathrm{L} / \mathrm{H}, \mathrm{L}-\mathrm{H}$, and $(\mathrm{L}-\mathrm{H}) /(\mathrm{L}+\mathrm{H})$, where $\mathrm{L}$ denotes lesion and $\mathrm{H}$ denotes healthy.

Then the normalized features were ranked based on the product of the Fisher distance and the area under the receiver operating characteristic (ROC) curve. The Fisher distance provides a measure of separability between classes, which is defined as FD $=\left|\mu_{b}-\mu_{c}\right| / \sqrt{\sigma_{b}{ }^{2}+\sigma_{c}^{2}}$, where $\mu_{b}$ and $\mu_{c}$ denote the class means and $\sigma_{b}$ and $\sigma_{c}$ the class variances. The ROC curve describes the relationship between the true positives and the false positives for several threshold values on a scatterplot. The abscissa of a ROC curve is termed 1 specificity, and its ordinate is sensitivity. Sensitivity indicates the fraction of correctly identified positive cases among all positive cases. Specificity indicates the fraction of correctly identified negative cases among all negative cases (benign lesions). Hence 1 specificity indicates the false-positive rate. The area under the ROC curve is a reflection of how effective the feature is at distinguishing between the classes. A ROC area close to 1 indicates good differentiation between benign and cancerous tissues. 
Table 1. Effective Image Features for Group 1 Lesions

\begin{tabular}{lllr}
\hline \multicolumn{1}{c}{ Image Feature ${ }^{a}$} & Statistic & Wavelength & \\
\hline Morlet wavelet, fiber 6 & Kunge (nm) & FD $^{*}$ ROCA $^{c}$ \\
Meyer wavelet, fiber 4 & Skewness & $625-742$ & 1.8979 \\
Meyer wavelet, fiber 5 & Kurtosis & $665-721$ & 1.8795 \\
Meyer wavelet, fiber 9 & Skewness & $664-722$ & 1.8482 \\
Morlet wavelet, fiber 9 & Skewness & $655-726$ & 1.7321 \\
Meyer wavelet, fiber 5 & Skewness & $663-722$ & 1.7317 \\
Meyer wavelet, fiber 4 & Kurtosis & $710-716$ & 1.6924 \\
Morlet wavelet, fiber 5 & Kurtosis & $626-741$ & 1.6277 \\
Meyer wavelet, fiber 3 & Skewness & $644-654$ & 1.6269 \\
Meyer wavelet, fiber 11 & Kurtosis & $641-691$ & 1.5664 \\
Meyer wavelet, fiber 10 & Skewness & $712-716$ & 1.5413 \\
Morlet wavelet, fiber 8 & Kurtosis & $622-745$ & 1.5343 \\
Meyer wavelet, fiber 8 & Kurtosis & $693-718$ & 1.5311 \\
\hline
\end{tabular}

${ }^{a} \mathrm{CWT}$ for all cases.

${ }^{b}$ Kurtosis is the fourth central moment divided by the fourth power of the standard deviation. Skewness is the third central moment divided by the cube of the standard deviation.

${ }^{c}$ Product of the Fisher distance and the area under the receiver operating characteristic curve.

We used a genetic algorithm (GA) to find the most effective combination of extracted wavelet features. This GA is capable of searching a high-dimensional feature space efficiently. Basically, the GA maximizes a fitness function that corresponds to the product of the Fisher distance and the area under the ROC curve in our case by carrying out three stochastic types of searching operation: population, crossover, and mutation. A chromosome set consisting of various points in the search space is initially set up. Then the population operation is applied to make clones of the chromosomes from a previous genera- tion. The number of chromosomes in the new generation is determined by the fitness value of the chromosome set in the old generation. Next, we perform a crossover operation to select two chromosomes at random. Random portions of these two selected chromosomes are chosen and swapped. Finally, the mutation operation is applied to invert random bits within the chromosomes. More details of GAs can be found in Ref. 10 .

Tables 1 and 2 list the features selected by the GA for groups 1 and 2, respectively. In these tables, skewness means the third central moment divided by the cube of the standard deviation. Kurtosis means the fourth central moment divided by the fourth power of the standard deviation. An Nth central moment is the expected value of $[x-\operatorname{mean}(x)]^{N}$.

\section{B. Feature Conditioning and Classification}

A feature vector was formed based on the effective wavelet features listed in Tables 1 and 2. Before feeding the feature vectors into a classifier, as reported in Ref. 7, we processed them to remove their correlations and reduce their dimensionality to one dimension. We removed the correlation by subtracting the mean feature vector and multiplying the feature vectors by the matrix $A_{1}=\left(\mathbf{e}_{1}, \mathbf{e}_{2}, \ldots, \mathbf{e}_{n}\right)^{t}$, where $\mathbf{e}_{i}(i=1,2 \ldots n)$ denotes an eigenvector of the covariance matrix. To reduce the dimensionality, the between-class scatter matrix $\left(S_{B}\right)$ and the within-class scatter matrix $\left(S_{w}\right)$ were calculated. For more details on the calculation of these matrices, refer to Ref. 11. We then reduced the dimensionality by minimizing the separability measure $J=$ Trace $\left(S_{w}{ }^{-1} S_{B}\right)$, which meant multiplying the feature vectors by the eigenvector corresponding to the non-

Table 2. Effective Image Features for Group 2 Lesions

\begin{tabular}{llll}
\hline \multicolumn{1}{c}{ Image Feature $^{a}$} & Wavelength & Range (nm) & FD*ROCA $^{c}$ \\
\hline Gaussian 2 wavelet, fiber 11 & Statistic ${ }^{b}$ & $587-673$ & 1.0081 \\
Gaussian 1 wavelet, fiber 6 & Skewness & $533-746$ & 0.9684 \\
Mexican hat wavelet, fiber 8 & Skewness & $543-547$ & 0.9388 \\
Gaussian 2 wavelet, fiber 10 & Variance & $577-673$ & 0.9336 \\
Gaussian 2 wavelet, fiber 9 & Skewness & $577-663$ & 0.9114 \\
Mexican hat wavelet, fiber 8 & Skewness & $548-552$ & 0.8928 \\
Gaussian 4 wavelet, fiber 10 & Energy & $529-586$ & 0.8727 \\
Gaussian 1 wavelet, fiber 8 & Kurtosis & $519-712$ & 0.8569 \\
Gaussian 2 wavelet, fiber 6 & Skewness & $567-717$ & 0.8486 \\
Gaussian 4 wavelet, fiber 10 & Skewness & $529-586$ & 0.8137 \\
Mexican hat wavelet, fiber 7 & Skewness & $529-562$ & 0.8119 \\
Gaussian 1 wavelet, fiber 4 & Variance & $543-736$ & 0.8073 \\
Morlet wavelet, fiber 4 & Skewness & $553-692$ & 0.7949 \\
Gaussian 4 wavelet, fiber 7 & Skewness & $698-736$ & 0.784 \\
Gaussian 4 wavelet, fiber 9 & Kurtosis & $524-586$ & 0.7804 \\
Morlet wavelet, fiber 4 & Kurtosis & $572-688$ & 0.7766 \\
Gaussian 4 wavelet, fiber 5 & Skewness & $698-736$ & 0.7607 \\
Gaussian 4 wavelet, fiber 8 & Kurtosis & $703-736$ & 0.7579 \\
Meyer wavelet, fiber 11 & Kurtosis & $524-605$ & 0.7436 \\
\hline
\end{tabular}

${ }^{a} \mathrm{CWT}$ for all cases.

${ }^{b}$ Skewness and Kurtosis as defined in Table 1.

${ }^{c}$ Product of the Fisher distance and the area under the receiver operating characteristic curve. 
zero eigenvalue of the matrix $\left(S_{w}{ }^{-1} S_{B}\right)$. Noting that this was a two-class problem, we reduced each feature vector to one dimension. In other words, all the effective wavelet features were linearly combined into one feature that generated the same separability measure $J$. Here this feature is referred to as the combined image feature (CIF). This combined single feature optimally represents, and is sensitive to, the spatiospectral differences between the measured data of benign and cancerous or dysplastic tissues.

As in Ref. 7, a bootstrap-based Bayes classifier was then used for each group, based on the selected features for that group. The Bayes decision rule for the minimization of the probability of error is as follows: for a feature vector $\mathbf{x}$, decide benign if $P($ benign $/ \mathbf{x})>$ $P$ (cancerous or dysplastic/x); otherwise decide cancerous or dysplastic. Using our one-dimensional CIF and assuming a Gaussian distribution, simplifies the decision rule to the following: decide benign if $g$ (benign) $>g$ (cancerous or dysplastic); otherwise, decide cancerous or dysplastic, where $g$ (benign) = $-\ln \left(\sigma_{b}\right)-\left(x_{\mathrm{CIF}}-\mu_{b}\right)^{2} / \sigma_{b}$ and $g($ cancerous or dysplastic $)=-\ln \left(\sigma_{c}\right)-\left(x_{\mathrm{CIF}}-\mu_{c}\right)^{2} / \sigma_{c}$ and $\mu_{b}$ and $\mu_{c}$ denote the class means and $\sigma_{b}$ and $\sigma_{c}$ the class variances. The bootstrap, which is a statistical method for estimating the sampling distribution of a statistic from the sample data, ${ }^{12}$ itself is used because of the limited number of samples. The bootstrap principle requires the following steps. Step 1: resampling. Using a pseudorandom number generator, draw a random sample of $m$ values, with replacement from observations $x$. Step 2: Calculation. Calculate the bootstrap estimate. Step 3: Repetition. Repeat steps 1 and 2 a large number of times to obtain a total of bootstrap estimates. Step 4: Approximate the distribution. ${ }^{12}$

We trained and tested the classifiers, using the data from 65 lesions (23 from group 1 and 42 from group 2), which corresponded to a total of 584 images. For this design dataset, classification rates (accuracies) of $100 \%$ for group 1 and $97 \%$ for group 2 were achieved (Fig. 4). The sensitivities obtained were $100 \%$ and $96 \%$ for groups 1 and 2 , respectively. The specificity obtained was $100 \%$ for both groups. The outcome of the classifier is based on the histological reports for the training set. We realize that histology is imperfect and sometimes subjective. Therefore we intend to involve multiple pathologists in future studies to improve the accuracy of the gold standard.

\section{Blind-Study Results}

To validate the performance of the classifiers we carried out a prospective double-blind study that included the collection of 222 new spatiospectral images from 37 skin lesions (six images per case: three from the lesion site and three from the adjacent normal skin). These images were different from those collected as described above for the design part of the study. Neither the person collecting the data nor the person processing the data was made aware of the biopsy outcomes. Of the 37 skin lesions, 14
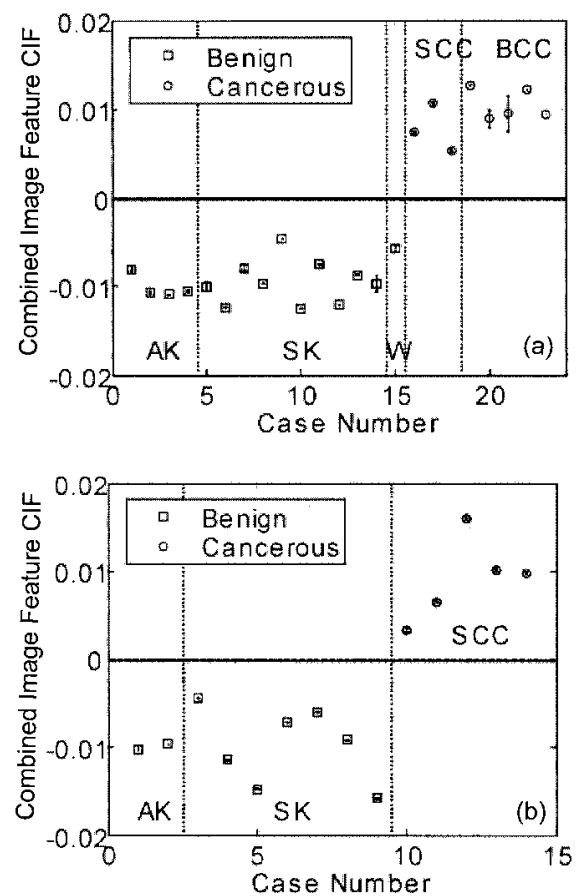

Fig. 4. (a) Results of the design dataset for group 1. (b) Prospective blind-study dataset results for group 1 . Vertical axes, CIF values; horizontal axes, relative case numbers.

belonged to group 1 and 23 to group 2 . The designed classifiers generated a classification rate of $100 \%$ for both of the blind-study groups, achieving $100 \%$ sensitivity and specificity (Fig. 5). Noting that it was obviously fortuitous that for group 2 the testing accuracy was better than the training accuracy, we carried out the following 50\%-50\% study to further validate the classifier's performance.

\section{D. $50 \%-50 \%$ Training and Testing}

We used $50 \%$ of the entire dataset, which included the design and blind-study datasets, for training and the remaining $50 \%$ for testing. Given the total of 37 lesions in group 1 (24 benign and 13), 12 benign and 7 cancerous lesions were randomly selected for training. Also, given the total of 65 lesions in group 2 (29 $\mathrm{CN}$ and $36 \mathrm{DN}$ ), we randomly selected 15 benign and 18 cancerous lesions for training. The remaining lesions were used for testing. This random selection for training and testing was repeated 10,000 times. For each repetition the classifier was redesigned without memory of the previous designs. As shown in Table 3, the average accuracies obtained were $100 \%$ for group 1 and $95 \%$ for group 2 .

\section{Physiological Parameters from Optical Properties}

This study was meant to provide insight into some of the physiological features of skin lesions that can be measured by SOIR. In keeping with this objective, we computed the absorption spectra $\left(\mu_{a}\right)$ and the reduced scattering spectra $\left(\mu_{s}{ }^{\prime}\right)$ and then used them to extract three independent physiological parameters: the concentration of oxygenated hemoglobin $\left(C_{\text {ox }}\right)$, 

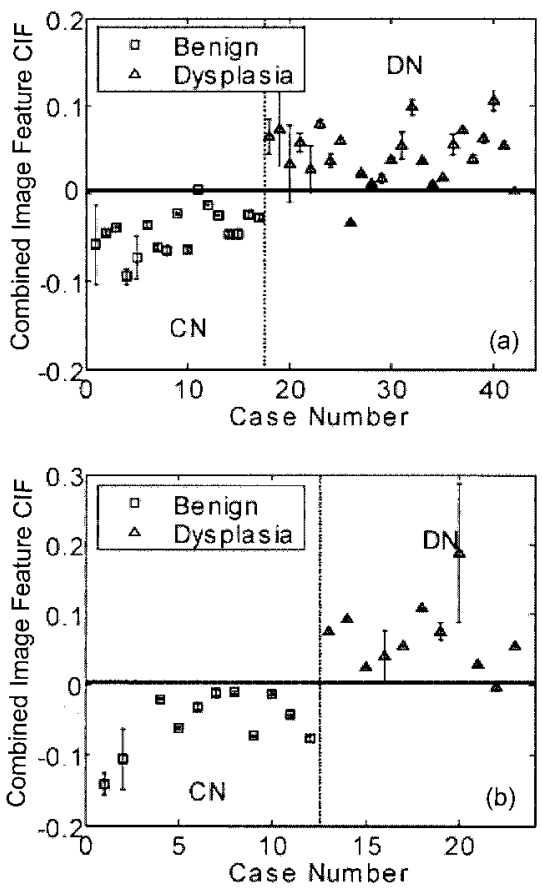

Fig. 5. (a) Results of the design dataset for group 1. (b) Prospective blind-study dataset results for group 1. Vertical axes, CIF values; horizontal axes, relative case numbers.

the concentration of deoxygenated hemoglobin $\left(C_{\mathrm{de}}\right)$, and the expected value $\langle\phi\rangle$ of the size distribution $f(\phi)$, where $\phi$ is the cell-nuclear size.

\section{A. Calculation of Absorption Coefficient and Reduced Scattering Coefficient}

Details of the calculation of absorption and reduced scattering coefficients by SOIR can be found in Ref. 8 . The absorption coefficient was calculated based on the diffusion theory of diffuse reflectance, whereas the calculation of the reduced scattering coefficient was based on an equation obtained for obliqueincidence reflectometry. ${ }^{13}$

Table 3. Average Accuracy, Sensitivity, and Specificity in the $50 \%-50 \%$ Analysis with 10,000 Random Selections

\begin{tabular}{lcc}
\hline \multicolumn{1}{c}{ Parameter } & $\begin{array}{c}\text { Lesion } \\
\text { Group } 1^{a}\end{array}$ & $\begin{array}{c}\text { Lesion } \\
\text { Group } 2^{a}\end{array}$ \\
\hline Average accuracy & 100 & 95.20 \\
Standard deviation, $\sigma_{\text {accuracy }}$ & 0 & 2.84 \\
Minimum accuracy & 100 & 87.50 \\
Maximum accuracy & 100 & 100 \\
Average sensitivity & 100 & 94.57 \\
Standard deviation, $\sigma_{\text {sensitivity }}$ & 0 & 3.89 \\
Minimum sensitivity & 100 & 88.89 \\
Maximum sensitivity & 100 & 100 \\
Average specificity & 100 & 96.01 \\
Standard deviation, $\sigma_{\text {specificity }}$ & 0 & 4.12 \\
Minimum specificity & 100 & 85.71 \\
Maximum specificity & 100 & 100 \\
\hline
\end{tabular}

${ }^{a}$ All parameter values are given in percentages.

\section{B. Extraction of Physiological Parameters}

Before extracting the physiological parameters we filtered the absorption spectra to reduce noise by using a linear-phase low-pass filter. We used the absorption spectra to extract a number of physiological parameters that were regarded as an integral part of the diagnostic optical signatures of the skin lesions or were reflective of their pathology. These parameters included the hemoglobin oxygen saturation and the concentration of hemoglobin, which are considered to be important parameters related to the disease state of lesions reflecting the presence of (1) angiogenesis, (2) lesion hemorrhage or hyperemia, and (3) lesion necrosis. ${ }^{14-16}$ The concentrations of oxygenated $\left(C_{\mathrm{ox}}\right)$ and deoxygenated hemoglobin $\left(C_{\mathrm{de}}\right)$ were calculated by solution of the following linear equation ${ }^{17}$ :

$$
\mu_{a}(\lambda)=\varepsilon_{\mathrm{ox}}(\lambda) C_{\mathrm{ox}}+\varepsilon_{\mathrm{de}}(\lambda) C_{\mathrm{de}}+\mu_{\mathrm{abg}},
$$

where $\mu_{a}\left[\mathrm{~cm}^{-1}\right]$ is the absorption coefficient; $\lambda$ is the wavelength; $\varepsilon_{\mathrm{ox}}\left[\mathrm{cm}^{-1}\right]$ and $\varepsilon_{\mathrm{de}}\left[\mathrm{cm}^{-1}\right]$ are the known extinction coefficients $\left[\mathrm{cm}^{-1} \mathrm{mM}^{-1}\right]$ of oxygenated and deoxygenated hemoglobin; and $\mu_{\mathrm{abg}}$ is the absorption coefficient caused by the local background tissue component, which was assumed to be wavelength insensitive. The oxygen saturation $\left(\mathrm{SO}_{2}\right)$ and the concentration $\left(C_{\mathrm{Hb}}\right)$ of total hemoglobin were computed based on the following equations: $\mathrm{SO}_{2}=$ $C_{\mathrm{ox}} /\left(C_{\mathrm{ox}}+C_{\mathrm{de}}\right)$ and $C_{\mathrm{Hb}}=C_{\mathrm{ox}}+C_{\mathrm{de}}$.

The reduced scattering spectra, $\mu_{s}^{\prime}$, were low-pass filtered likewise to reduce noise. The sizes of the scattering centers in cells are of the order of 100 $1000 \mathrm{~nm}$, sizes that are similar to the wavelengths of light in SOIR. We used the scattering spectra to calculate the size distribution of the optical scatterers, using inverse Mie theory.4,18,19 This was done based on the following relationship:

$$
\mu_{s}{ }^{\prime}(\lambda)=C_{s} \int_{0}^{\infty} \frac{3 Q_{s}(\phi, n, \lambda)[1-g(\phi, n, \lambda)]}{2 \phi} f(\phi) \mathrm{d} \phi,
$$

where $\mu_{s}{ }^{\prime}(\lambda)$ is the reduced scattering spectrum of the tissue, $C_{s}$ is the total volume concentration of the scatterers, $Q_{s}($ ) is the scattering efficiency, $\phi$ is the diameter of the scatterers, $n$ is the relative index of refraction of the scatterers, $\lambda$ is the wavelength, $g()$ is the scattering anisotropy, and $f()$ is the size distribution-assumed to be a Rayleigh distribution. We used Mie theory to calculate $Q_{s}()$ and $g($ ).20

\section{Physiological Parameter Results}

The absorption spectra were extracted for 26 of the total lesions, and reduced scattering spectra were extracted for 51 of the total lesions because the remaining lesions generated unreliable spectral fits, possibly because of the heterogeneity of the lesions. Table 4 lists the average and the standard deviation for each physiological parameter in each group into which we had divided the lesions and for each class: the oxygen saturation $\left(\mathrm{SO}_{2}\right)$, the concentration of he- 
Table 4. Averages of Physiological Parameters for Each Lesion Group

\begin{tabular}{lccccc}
\hline & \multicolumn{2}{c}{ Group 1 } & & \multicolumn{2}{c}{ Group 2 } \\
\cline { 2 - 3 } Parameter & Cancerous Lesions & Benign Lesions & & DN Lesions & Benign Lesions \\
\hline $\mathrm{SO}_{2}$ & $0.46 \pm 0.02$ & $0.49 \pm 0.02$ & & $0.44 \pm 0.04$ & $0.48 \pm 0.06$ \\
$C_{\mathrm{Hb}}(/ \mathrm{mM})$ & $0.14 \pm 0.08$ & $0.31 \pm 0.21$ & & $0.60 \pm 0.69$ & $0.80 \pm 0.34$ \\
$\phi(\mu \mathrm{m})$ & $20.4 \pm 7.5$ & $6.3 \pm 5.5$ & & $15.7 \pm 8.8$ & $9.0 \pm 4.8$ \\
\hline
\end{tabular}

moglobin $\left(C_{\mathrm{Hb}}\right)$, and the expected value of the size distribution $f(\phi)$ in micrometers. We are developing a new algorithm with which to extract the optical properties from more lesions such that statistically significant differences between the benign and the cancerous or dysplastic groups can be identified.

\section{Physiological Explanation of Classification Outcomes}

Table 4 summarizes the results for the physiological parameters extracted from the optical properties of the tissues that we examined. This table shows that the cancerous lesions have a lower level of oxygen saturation than do the benign lesions. The lower oxygen saturation in cancer lesions may be related to several factors, including (1) tumor death and necrosis, (2) intratumoral hemorrhage with blood stagnation, (3) abnormal blood supply and distribution, and (4) metabolic abnormalities. ${ }^{15}$ This table also shows that cancerous and DN lesions tend to have lower concentrations of blood. Although angiogenesis should increase the concentration of blood, the volume-averaged concentration of total hemoglobin $C_{\mathrm{Hb}}$ in cancerous or $\mathrm{DN}$ lesions suggests that cell death probably dominates angiogenesis in skin lesions. Table 4 indicates that the expected value of size distribution $f(\phi)$ in the malignant lesions is larger than that in the benign lesions. Cell nuclei, mitochondria, other cytoplasmic organelles, and structures within cell nuclei are expected to be significant light scatterers, and the average effective size of the scattering centers is of the order of a few hundred nanometers. ${ }^{21-24}$ However, as indicated by the results, the expected value of the size distribution reflects primarily the sizes of cell nuclei. Backman and co-workers found that the average sizes of the optical scatterers correlate well with the sizes of cell nuclei.4,25 Although each physiological parameter produces a general trend in each type of lesion, biological variability prevents accurate diagnosis of malignancy by use of a single physiological parameter; however, a CIF that is based on the combination of multiple physiological parameters does provide a highly accurate diagnostic indicator.

\section{Conclusions}

This paper represents an extension of a study classifying skin lesions by spectroscopic oblique-incidence reflectometry. An important characteristic of the classification scheme is that one requires no assumptions to produce a successful classification outcome. In the prospective double-blind study, the image analysis system that we developed achieved a successful classification rate of $100 \%$ for both groups. In the 50\%-50\% rotation study, the average accuracy obtained was $100 \%$ for group 1 and $95 \%$ for group 2 . These results are highly promising and indicate that the approach presented here can potentially be used by dermatologists for computer-assisted classification of skin lesions.

The authors acknowledge the assistance of and helpful discussions with M. Mehrubeoglu, S. Thomsen, and J. Mourant. The research reported here was sponsored in part by National Institutes of Health grant R01 CA71980, National Science Foundation grant BES-9734491, and Texas Higher Education Coordinating Board grant 000512-0063-2001.

\section{References}

1. American Cancer Society, "Melanoma and non-melanoma skin cancer," http://www.cancer.org (2002).

2. H. Ganster, A. Pinz, R. Rohrer, E. Wildling, M. Binder, and H. Kittler, "Automated melanoma recognition," IEEE Trans. Med. Imaging 20, 233-239 (2001).

3. V. P. Wallace, D. C. Crawford, P. S. Mortimer, R. J. Ott, and J. C. Bamber, "Spectrophotometric assessment of pigmented skin lesions: methods and feature selection for evaluation of diagnostic performance," Phys. Med. Biol. 45, 735-751 (2000).

4. V. Backman, M. B. Wallace, L. T. Perelman, J. T. Arendt, R. Gurjar, M. G. Muller, Q. Zhang, G. Zonios, E. Kline, T. McGillican, S. Shapshay, T. Valdez, K. Badizadegan, J. M. Crawford, M. Fitzmaurice, S. Kabani, H. S. Levin, M. Seiler, R. R. Dasari, I. Itzkan, J. Van Dam, and M. S. Feld, "Detection of preinvasive cancer cells," Nature 406, 35-36 (2000).

5. K. M. Cooney, K. W. Gossage, M. J. McShane, E. W. J. van der Breggen, M. Motamedi, and G. L. Cote, "Development of an optical system for the detection of oral cancer using nearinfrared spectroscopy," in Proceedings of the 20th Annual International Conference of the IEEE Engineering in Medicine and Biology Society (Institute of Electrical and Electronics Engineers, Piscataway, N.J. (1998), pp. 906-909.

6. J. Roning and M. Riech, "Registration of nevi in successive skin images for early detection of melanoma," in Proceedings of the Fourteenth International Conference on Pattern Recognition (Institute of Electrical and Electronics Engineers, Piscataway, N.J., (1998), pp. 352-357.

7. M. Mehrubeoglu, N. Kehtarnavaz, G. Marquez, M. Duvic, and L.-H. Wang, "Skin lesion classification using oblique-incidence diffuse reflectance spectroscopic imaging," Appl. Opt. 41, 182192 (2002)

8. G. Marquez and L.-H. Wang, "White light oblique incidence reflectometer for measuring absorption and reduced scattering spectra of tissue-like turbid media," Opt. Express 1, 454-460 (1997), http://www.opticsexpress.org.

9. J. Groswami and A. Chan, Fundamentals of Wavelets, Theory, Algorithms and Applications, 1st ed. (Wiley Interscience, New York, 1999). 
10. H. Vafaie and K. Jong, "Genetic algorithms as a tool for feature selection in machine learning," in Proceedings of the IEEE International Conference on Tools with Artificial Intelligence (Institute of Electrical Engineers, Piscataway, N.J., 1992), pp. 200-203.

11. R. O. Duda, P. E. Hart, and D. G. Stork, Pattern Classification, 2nd ed. (Wiley Interscience, New York, 2000).

12. A. M. Zoubir and B. Boashash, "The bootstrap and its applications in signal processing," IEEE Signal Process. Mag. 15(1), 56-76 (1998).

13. L.-H. Wang and S. L. Jacques, "Use of a laser beam with an oblique angle of incidence to measure the reduced scattering coefficient of a turbid medium," Appl. Opt. 34, 2362-2366 (1995).

14. H. B. Stone, J. M. Brown, T. L. Phillips, and R. M. Sutherland, "Oxygen in human tumors: correlations between methods of measurement and response to therapy," Radiat. Res. 136, 422434 (1993).

15. R. S. Cotran, V. Kumar, and T. Collins, Robbins Pathologic Basis of Disease, 6th ed. (Saunders, Philadelphia, Pa., 1999), pp. 1-498 and 1170-1268.

16. S. Thomsen and D. Tatman, "Physiological and pathological factors of human breast disease that can influence optical diagnosis,” Ann. N. Y. Acad. Sci. 838, 171-193 (1998).

17. H. Liu, D. A. Boas, Y. Zhang, A. G. Yodh, and B. Chance, "Determination of optical properties and blood oxygenation in tissue using continuous NIR light," Phys. Med. Biol. 40, 19831993 (1995).

18. H. Jiang, G. Marquez, and L.-H. Wang, "Particle sizing in con- centrated suspensions by use of steady-state, continuous-wave photon migration techniques," Opt. Lett. 23, 394-396 (1998).

19. E. Sevick-Muraca, J. Pierce, H. B. Jiang, and J. Kao, "Photonmigration measurement of latex size distribution in concentrated suspensions," AIChE J. 43, 655-664 (1997).

20. H. C. van de Hulst, Light Scattering by Small Particles (Dover, New York, 1981).

21. J. R. Mourant, J. P. Freyer, A. H. Hielscher, A. A. Eick, D. Shen, and T. M. Johnson, "Mechanisms of light scattering from biological cells relevant to noninvasive optical-tissue diagnostics," Appl. Opt. 37, 3586-3593 (1998).

22. J. R. Mourant, M. Canpolat, C. Brocker, O. Esponda-Ramos, T. M. Johnson, A. Matanock, K. Stetter, and J. P. Freyer, "Light scattering from cells: the contribution of the nucleus and the effects of proliferative status," J. Biomed. Opt. 5, 131137 (2000).

23. R. Drezek, A. Dunn, and R. Richards-Kortum, "Light scattering from cells: finite-difference time-domain simulations and goniometric measurements," Appl. Opt. 38, 3651-3661 (1999).

24. J. R. Mourant, T. M. Johnson, V. Doddi, and J. P. Freyer, "Angular dependent light scattering from multicellular spheroids," J. Biomed. Opt. 7, 93-99 (2002).

25. L. T. Perelman, V. Backman, M. Wallace, G. Zonios, R. Manoharan, A. Nusrat, S. Shields, M. Seiler, C. Lima, T. Hamano, I. Itzkan, J. Vandam, J. M. Crawford, and M. S. Feld, "Observation of periodic fine structure in reflectance from biological tissue-a new technique for measuring nuclear size distribution," Phys. Rev. Lett. 80, 627-630 (1998). 